\title{
Antitumor effects of a drug combination targeting glycolysis, glutaminolysis and de novo synthesis of fatty acids
}

\author{
DIANA CERVANTES-MADRID $^{1}$ and ALFONSO DUEÑAS-GONZÁLEZ ${ }^{2}$ \\ ${ }^{1}$ Division of Basic Research, Instituto Nacional de Cancerología; ${ }^{2}$ Instituto de Investigaciones Biomédicas, Universidad \\ Nacional Autónoma de México (UNAM)/Instituto Nacional de Cancerología, Mexico City, Tlalpan 14080, Mexico
}

Received April 8, 2015; Accepted May 22, 2015

DOI: $10.3892 /$ or.2015.4077

\begin{abstract}
There is a strong rationale for targeting the metabolic alterations of cancer cells. The most studied of these are the higher rates of glycolysis, glutaminolysis and de novo synthesis of fatty acids (FAs). Despite the availability of pharmacological inhibitors of these pathways, no preclinical studies targeting them simultaneously have been performed. In the present study it was determined whether three key enzymes for glycolysis, glutaminolysis and de novo synthesis of FAs, hexokinase-2, glutaminase and fatty acid synthase, respectively, were overexpressed as compared to primary fibroblasts. In addition, we showed that at clinically relevant concentrations lonidamine, 6-diazo-5-oxo-L-norleucine and orlistat, known inhibitors of the mentioned enzymes, exerted a cell viability inhibitory effect. Genetic downregulation of the three enzymes also reduced cell viability. The three drugs were highly synergistic when administered as a triple combination. Of note, the cytotoxicity of the triple combination was low in primary fibroblasts and was well tolerated when administered into healthy BALB/c mice. The results suggest the feasibility and potential clinical utility of the triple metabolic targeting which merits to be further studied by using either repositioned old drugs or newer, more selective inhibitors.
\end{abstract}

\section{Introduction}

There is a strong rationale for the 'complete' blockade of the malignant metabolic phenotype as antitumor therapy. It is currently widely accepted that the 'malignant metabolic phenotype' is another hallmark of cancer and that it results from a number of gain-of-function mutations in oncogenes and loss-of-function of tumor-suppressor genes $(1,2)$. This malig-

Correspondence to: Dr Alfonso Dueñas-González, Instituto de Investigaciones Biomédicas, Universidad Nacional Autónoma de México (UNAM)/Instituto Nacional de Cancerología, 22 San Fernando, Sección XVI, Mexico City, Tlalpan 14080, Mexico

E-mail: alfonso_duenasg@yahoo.com

Key words: cancer metabolism, glycolysis, synthesis of fatty acids, glutaminolysis, drug repositioning nant metabolic phenotype is characterized by higher rates of glycolysis, higher rates of glutaminolysis and an increased de novo synthesis of fatty acids (FAs) or lipogenic phenotype.

Malignant cell proliferation requires energy in the form of ATP as well as synthesis of macromolecules including nucleotides, amino acids and lipids. For most mammalian cells in culture, the only two molecules catabolized in appreciable quantities are glucose and glutamine. This means that glucose and glutamine supply most of the carbon and nitrogen for the synthesis of macromolecules, energy and reducing equivalents necessary to support cell growth (3-5).

A third metabolic characteristic of the malignant phenotype is increased lipogenesis and this process requires glucose and glutamine. Most adult tissues take up circulating FAs instead of using de novo synthesis $(6,7)$. During malignant transformation, cells have been shown to upregulate FA synthesis instead of increasing the uptake of exogenous FAs to meet the increasing demand for biomass production. This process is thought to be part of a general metabolic remodeling from a non-proliferative catabolic phenotype to a proliferative anabolic phenotype. During this process, glucose is first converted to acetyl-CoA in the mitochondrial matrix and used to synthesize citrate in the tricarboxylic acid (TCA) cycle. Glutamine appears to be critical for lipid synthesis as it supplies carbon in the form of mitochondrial oxaloacetate to maintain citrate production in the first step of the TCA cycle. Thus, the metabolism of glutamine and glucose is orchestrated to support the production of acetyl-CoA and NADPH needed for FA synthesis $(8,9)$.

Despite the strong rationale for developing a combination of drugs to simultaneously target these three key processes for malignant cells, there is no experimental evidence available thus far to support this hypothesis despite the availability of drugs known to inhibit glycolysis, glutaminolysis and the de novo FA synthesis. In this regard, among anti-glycolytic drugs, lonidamine, an inhibitor of hexokinase-2 (HK-2) was clinically used but was deemed ineffective (10). Similarly, 6-diazo-5-oxo-L-norleucine (DON) is a well-known inhibitor of the enzyme glutaminase (GLS) that was also clinically tested and deemed to be ineffective (11). Regarding FA synthesis, a number of experimental compounds have been developed, none of which has been utilized in the clinic (12). Of these, orlistat has been extensively evaluated in vitro and in vivo and has shown promising activity in a number of malignancies due 
to its ability to inhibit FA synthase, the gene product of fatty acid synthase (FASN), responsible for the de novo synthesis of FAs (13-22).

These above mentioned findings provide strong support to determine whether a combination of inhibitors of these three key pathways of cancer cell metabolism exert higher antitumor effects as compared to inhibition of each pathway individually. The results of this study showed that the combination of lonidamine, DON and orlistat, inhibitors of glycolysis, glutaminolysis and de novo synthesis of FAs, respectively, exhibits significant efficacy in vitro and is tolerable in vivo.

\section{Materials and methods}

Cell lines, drugs and antibodies. Thirteen human malignant cell lines were used for the drug treatments: Prostate (DU-145 and PC-3), ovarian (SK-OV-3) and breast (MDA-MB-231 and MCF7) cancer, osteosarcoma (U-2OS), cervical (HeLa), gastric (AGS), glioma (D54), bladder (T24), melanoma (A-375), pancreatic (PANC-1) and colon (SW480) cancer. The cell lines were obtained from the American Type Culture Collection (ATCC; Manassas, VA, USA). Primary lung fibroblasts from a non-cancer individual were kindly provided by Dr Moisés Selman (National Institute for Respiratory Diseases, Mexico City, Mexico) (23). The cells were cultured in Dulbecco's modified Eagle's medium-F12 (cat. no. 12400-016) supplemented with $10 \%$ fetal bovine serum (FBS) (cat. no. 16000-044) with the exception of fibroblasts which were cultured in F-12 medium (cat. no. 21127-022) (all from Gibco-Life Technologies, Grand Island, NY, USA) supplemented with 5\% FBS. The cells were maintained with $5 \% \mathrm{CO}_{2}$ in a humidified incubator at $37^{\circ} \mathrm{C}$. Lonidamine (cat. no. 1646) and DON (cat. no. D2145-25MG) were purchased from Tocris Bioscience (Bristol, UK) and Sigma-Aldrich (St. Louis, MO, USA), respectively. Orlistat was a gift from Psicofarma, S.A. de C.V. (Mexico City, Mexico).

Drug solutions. Lonidamine was dissolved in dimethyl sulfoxide (DMSO; cat. no. D2650), DON in culture medium without serum and orlistat in ethanol (cat. no. E7023) (all from Sigma-Aldrich). Stock solutions were prepared and stored at $4^{\circ} \mathrm{C}$ for up to one week. Appropriate dilutions from the stock solutions were produced in medium before adding drugs to the experimental cultures. The final concentration of DMSO and ethanol in growth medium for all the drug treatments was maintained at $<0.1 \%$.

Cell line treatments. For the cell viability assays, $1 \times 10^{5}$ cells were seeded in 6-well plates and allowed to attach to the bottom overnight and then treated with $500 \mu \mathrm{M}$ DON, $100 \mu \mathrm{M}$ lonidamine, $25 \mu \mathrm{M}$ orlistat and a combination of the three drugs at the same concentration as the one used for single drug treatment for $48 \mathrm{~h}$. The concentrations for the three drugs were selected on the information that these were clinically achievable according to the literature. Thus, all the cell lines were treated with the same concentration of the drugs. Vehicle-treated cells were used as controls. Treated cells were stained with $0.4 \%$ trypan blue solution and counted under an inverted microscope with a Neubauer chamber. Any cells that excluded the dye were considered as viable. Experiments were performed in triplicate, with duplicates for each experiment.
$R T-q P C R$. Total RNA isolation from untreated cell lines was carried out using TRIzol (cat. no. 15596-018; Invitrogen Life Technologies, Carlsbad, CA, USA) and reverse transcription was performed from $1 \mu \mathrm{g}$ of total RNA using random primers and MultiScribe Reverse Transcriptase (cat. no. 4304134; Applied Biosystems, Foster City, CA, USA), according to the manufacturer's instructions. $2 \mu \mathrm{g}$ cDNA was used for the amplification of HK-2, GLS and FASN by RT-PCR with Bio-Rad iCycler iQ Real-Time PCR detection system (Bio-Rad, Hercules, CA, USA). $\beta$-actin mRNA levels were used for normalization of the interest genes. HK-2, GLS and FASN qPCRs were run using primers and iQ SYBR-Green Supermix (cat. no. 170-8882; Bio-Rad), whereas $\beta$-actin qPCR was run using TaqMAn probe and $2 \mathrm{X}$ TaqMan Universal PCR Master mix (cat. no. 4304437; Applied Biosystems). PCR reactions were run in triplicate and repeated in three independent experiments. The standard curve method for absolute quantification was used to calculate the gene copy number relative to $\beta$-actin mRNA expression levels. The final primer concentration for HK-2 was $0.5 \mu \mathrm{M}$ for GLS was $0.4 \mu \mathrm{M}$ for FASN was $0.5 \mu \mathrm{M}$ and for $\beta$-actin probe was $0.1 \mu \mathrm{M}$. PCR conditions used were: AmpErase UNG incubation for $2 \mathrm{~min}$ at $50^{\circ} \mathrm{C}$ ( $\beta$-actin); polymerase activation for $10 \mathrm{~min}$ at $95^{\circ} \mathrm{C}$ (HK-2, GLS, FASN and $\beta$-actin) and denaturation for $30 \mathrm{sec}$ at $95^{\circ} \mathrm{C}\left(\mathrm{HK}-2, \mathrm{GLS}\right.$ and FASN) and $15 \mathrm{sec}$ at $95^{\circ} \mathrm{C}(\beta$-actin); annealing for $30 \mathrm{sec}$ at $60^{\circ} \mathrm{C}$ (HK-2, GLS and FASN) for $1 \mathrm{~min}$ at $60^{\circ} \mathrm{C}(\beta$-actin). Extension and cycle numbers were carried out as follows: $40 \mathrm{sec}$ at $72^{\circ} \mathrm{C}, 40$ cycles (GLS and FASN); $40 \mathrm{sec}$ at $72^{\circ} \mathrm{C}, 35$ cycles (HK-2) and 40 cycles ( $\beta$-actin). The primer pairs used were: GLS forward, 5'-GGAC AGAGGCATTCTACTG-3' and reverse, 5'ATCTTAGTCCAC TCGGCTC-3'; HK-2 forward, 5'-GAGAGGGGACTTTGA TATC-3' and reverse, 5'GGCGTTGCTGCCCGTGCC-3'; FASN forward, 5'-CATCCAGATAGGCCTCATAGAC-3' and reverse, 5'-CTCCATGAAGTAGGAGTGGAA-3'. The probe for $\beta$-actin was selected from Life Technology pre-designed probes, Hs 01060665_g1 TaqMan Probe.

Immunofluorescence (IF). SW480 cells were seeded in 4-well chambers (Lab-Tek II Chamber Slide system; Thermo Fisher Scientific, Inc., Waltham, MA, USA) and allowed to adhere overnight at $37^{\circ} \mathrm{C}$ with $5 \% \mathrm{CO}_{2}$. The cells were fixed with $2 \%$ paraformaldehyde for $20 \mathrm{~min}$ and permeabilized with $0.01 \%$ Triton X-100 for $30 \mathrm{sec}$ and blocked with power block universal blocking reagent 10X (cat. no. BS-1310-25; Biogenex, Fremont, CA, USA) for $1 \mathrm{~h}$. Incubation was performed overnight at $4^{\circ} \mathrm{C}$ with the following antibodies and concentrations: mouse monoclonal raised against recombinant GLS-K of human origin (cat. no. IJ-2) at $1,000 \mathrm{ng} / \mathrm{ml}$; goat polyclonal raised against a peptide mapping at the C-terminal of HK-2 of human origin (cat. no. C-14) at $800 \mathrm{ng} / \mathrm{ml}$; or rabbit polyclonal antibody raised against amino acids 2205-2504 mapping at the C-terminal of FASN of human origin (cat. no. H-300) at $400 \mathrm{ng} / \mathrm{ml}$. DyLight 488-conjugated affinipure bovine antigoat (1:200) was used to reveal HK-2; DyLight 649-conjugated affinipure donkey anti-rabbit (1:300) to detect FASN; and alexa fluor 594 goat anti-mouse $\operatorname{IgG}(\mathrm{H}+\mathrm{L})$ antibody (1:200) for GLS. Antibodies were diluted in 1\% BSA in PBS. Secondary antibody incubation was for $1 \mathrm{~h}$. UltraCruz Mounting Medium (Santa Cruz Biotechnology, Inc., Dallas, TX, USA) was used 
for nuclei staining after secondary antibody incubation and it contained 4',6-diamidino-2-phenylindole (DAPI). The slides were mounted with a coverslip. After each step, washing was performed with $0.05 \%$ PBS Tween-20. The slides were analyzed using a Zeiss Axioplan Epifluorescence microscope (Carl Zeiss, Inc., Göettingen, Germany). The image density was measured by ImageJ 1.440 . At least 30 cells were measured per experiment. The primary antibodies against HK-2, GLS-K and FASN were purchased from Santa Cruz Biotechnology, Inc. DyLight 488-conjugated affinipure bovine anti-goat and 649-conjugated affinipure donkey anti-rabbit were purchased from Jackson Immuno Research Laboratories (West Grove, PA, USA). Alexa Fluor 594 goat anti-mouse IgG (H+L) antibody was purchased from Invitrogen Life Technologies.

siRNA transfection. Specific siRNAs were used to knock down the expression of FASN, GLS and HK-2 genes. SW480 cells $\left(1 \times 10^{5}\right)$ were seeded in 6 -well plates to achieve $\sim 30 \%$ confluence at the time of transfection. Then, $100 \mathrm{pmol}$ Silencer select RNAi (HK-2 cat. no. 4390824 ID s6562, GLS cat. no. 4392420 ID s5838 and FASN cat. no. 4390824 ID s5032) were diluted in $200 \mu \mathrm{l}$ Opti-MEM I reduced serum medium (cat. no. 31985-070) (all from Ambion, Austin, TX, USA). Lipofectamine RNAiMAX transfection reagent $(2 \mu \mathrm{l})$ was diluted in $200 \mu$ l Opti-MEM I reduced serum medium. The two mixes were pooled and incubated for $20 \mathrm{~min}$ at room temperature. Silencer select negative control no. 1 siRNA was used as the negative control and had no significant similarity to human gene sequences and no significant effect on cell viability. The mix was added to each well containing the cells in $2 \mathrm{ml}$ Opti-MEM I reduced serum medium without serum and replaced after $8 \mathrm{~h}$ incubation by new fresh media or media plus drugs for the experiments combining siRNA and the drugs. The siRNA final concentration for the three genes and the negative control was $50 \mathrm{nM}$. The cells were collected for RNA extraction after counting using trypan blue dye exclusion.

Quantification of the synergism of lonidamine, DON and orlistat. SW480 $\left(1 \times 10^{6}\right)$ cells were seeded in a 6-well plate and treated for $48 \mathrm{~h}$ with different concentrations of lonidamine, DON and orlistat as monotherapy to establish their $\mathrm{IC}_{50}$ and in triple combination (lonidamine, DON and orlistat) at doses determined by the $\mathrm{IC}_{50}$ of each drug, while maintaining the constant ratio of 4:1:2 for lonidamine, DON and orlistat, respectively. Trypan blue dye exclusion assay was used to assess cell viability. To quantify the interaction of the three drugs, analyses were based on the combination index (CI). A $\mathrm{CI}$ of 1 indicated an additive effect between the agents, whereas a $\mathrm{CI}<1$ or $>1$ indicated synergism or antagonism, respectively. The Chou-Talalay method was used to analyze synergism, using CalcuSyn software (Biosoft, Cambridge, UK).

Administration of the triple combination in vivo. To determine whether the triple combination was tolerable in vivo, 6-week-old BALB/c female mice were obtained from Harlan Laboratories, Inc. (Indianapolis, IN, USA). Six mice/cage were housed with access to food and water ad libitum in a 12:12 light-dark cycle. Prior to treatment, the animals were allowed to acclimatize for 1 week. The animal studies were in compliance with policies of the Institutional Research Ethics Board and Animal Care Committee of the Instituto Nacional de Cancerología, Mexico (permit nos. CA006/CB595/10 and INCAN/CC/010/10). Six mice were injected with lonidamine $(0.125 \mathrm{mg} / \mathrm{kg})$ and orlistat $(240 \mathrm{mg} / \mathrm{kg})$ daily and DON $(0.250 \mathrm{mg} / \mathrm{kg})$ on days 1,4 and 8 for 3 weeks by intraperitoneal route (DON only three injections in the 21-day cycle). A control vehicle group of 6 animals was treated in an identical schedule. Animal injections were performed inside a laminar flow cabinet. Changes in body weight were used as a parameter to measure toxic effects. Each mouse was clinically inspected and weighed on days $0,7,14$ and 21 . At week 4, the mice were sacrificed in a $\mathrm{CO}_{2}$ chamber. All the experimental procedures performed aimed to reduce suffering and are in strict accordance with the Regional Animal Ethics Committee approval.

Statistical analysis. Data are presented as means \pm standard deviations (SD) from three determinations. Statistical differences between two groups were evaluated using the Student's t-test. The Pearson correlation test was used to analyze the correlation between mRNA and protein expression of the three enzymes. Statistical analyses were conducted using Excel 2008 for Mac (Microsoft Corp., Redmond, WA, USA) for the Student's t-test and SPSS statistics (SPSS, Inc., Chicago, IL, USA) for Pearson correlation

\section{Results}

Expression of the mRNA of $H K-2, G L S$ and FASN. The metabolic phenotype characterized by increased glycolysis, glutaminolysis and de novo synthesis of FAs is common to cancer cells. Thus HK-2, GLS and FASN, which are at least partly responsible for such phenotypes, must be expressed in most cancer cells. To demonstrate this, we used RT-qPCR to determine the expression levels of these genes in a panel of cancer cell lines. Fig. 1 shows that all the cell lines overexpressed HK-2 at variable degrees in comparison to normal primary fibroblasts. These differences were statistically significant, with exception of the pancreatic cancer cells (PANC-1). MCF7 and D54 had the highest expression. For GLS, all 13 malignant cell lines were overexpressed compared to fibroblasts. HeLa, DU 145 and T24 had the highest expression whereas MCF7, PC-3, PANC-1, SW480 and AGS had a lower expression with differences being statistically significant. FASN was also statistically significantly overexpressed in all the cell lines analyzed. Each of the three genes was minimally expressed in primary fibroblasts.

Protein expression of $H K-2, G L S-K$ and FASN. To investigate whether there was overexpression at protein level, the cells were analyzed by IF imaging. As shown in Fig. 2, there was a variable overexpression for all three gene products in the whole cell lines in comparison with the primary fibroblasts. The differences were statistically significant with the exception of GLS in the ovarian SK-OV-3 cell line compared to fibroblasts.

Correlation between $m R N A$ and protein expression levels of target genes. To determine whether there was a correlation between the levels of mRNA and protein in the malignant 

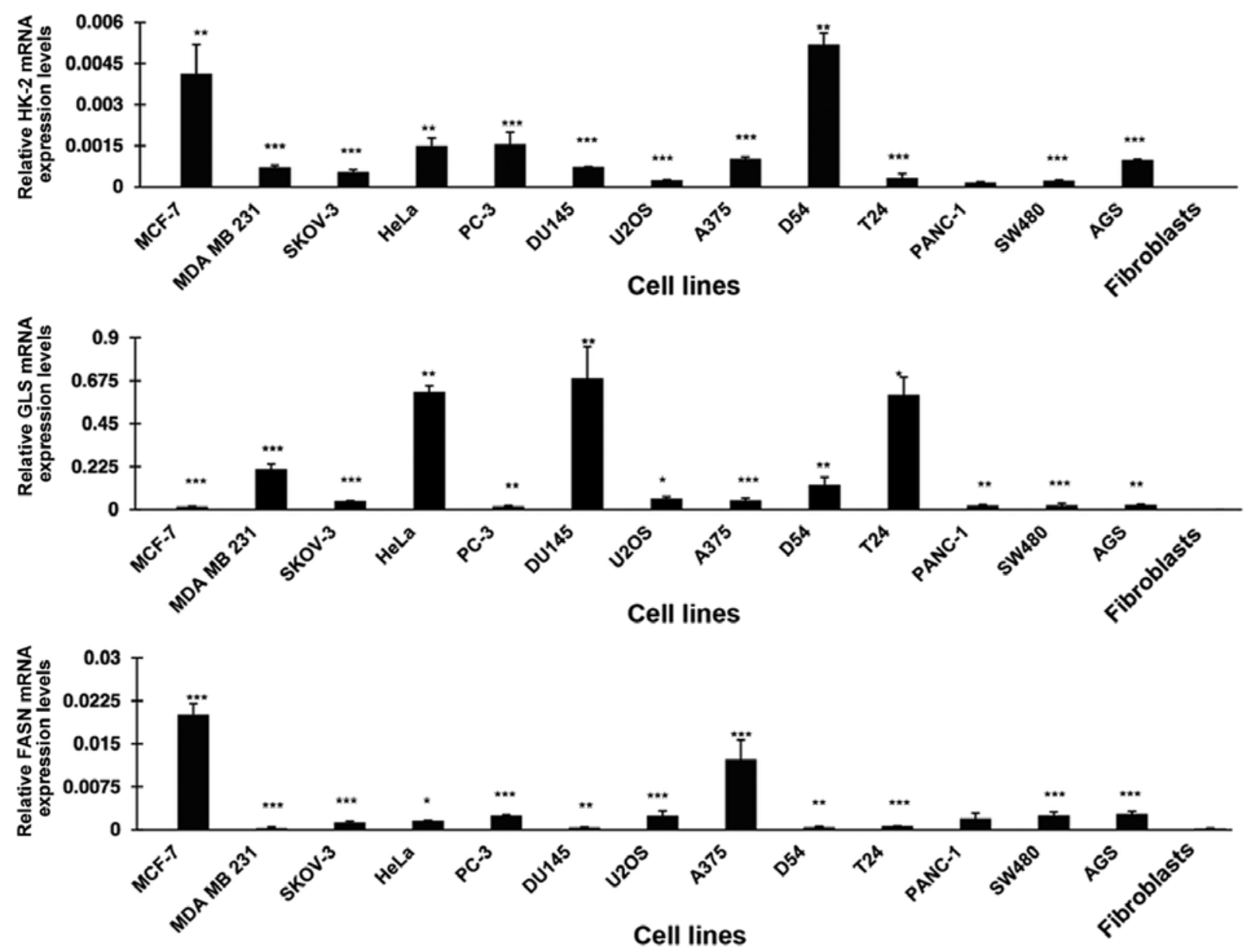

Figure 1. mRNA gene expression of HK-2, GLS and FASN in different cell lines. RT-qPCR was used to determine the basal mRNA expression levels of HK-2, GLS and FASN using the absolute quantification method. The mRNA expression levels are relative to $\beta$-actin mRNA levels. Data are presented as means \pm SD of three independent experiments performed in triplicate. Significant differences between fibroblasts and the different malignant cell lines are indicated as ${ }^{*} \mathrm{P}<0.05,{ }^{* *} \mathrm{P}<0.01$ and ${ }^{* * *} \mathrm{P}<0.001$. The three target genes were overexpressed in all the malignant cell lines as compared to the primary fibroblasts. HK-2, hexokinase-2; GLS, glutaminase; FASN, fatty acid synthase.

cell lines, a correlation analysis was performed. For the three enzymes there was no correlation between their protein and mRNA expression, GLS $(\mathrm{R}=0.055, \mathrm{P}=0.86), \mathrm{HK}-2(\mathrm{R}=0.487$, $\mathrm{P}=0.91)$ and FASN $(\mathrm{R}=0.56, \mathrm{P}=0.056)$.

Cell viability inhibition by lonidamine, DON and orlistat as single drugs. Once demonstrated that all cell lines expressed the target genes at RNA and protein levels, cell viability assays after treatments were performed and the results analyzed at 48 h. Fig. 3 shows that, as compared with primary fibroblasts, lonidamine led to a reduction in cell viability ranging from $56.7 \%$ for A-375 melanoma cells to $19.5 \%$ in SK-OV-3 ovarian cancer cells. The ovarian cancer cell line was the only one that did not show any statistically significant difference $(\mathrm{P}=0.16)$. Essentially no cell viability inhibition was observed for the primary fibroblasts. DON appeared to reduce viability to a greater extent as compared to lonidamine. For DON, the greatest inhibition was observed for D54 (81\%) and the lowest for T24 (35\%), with all values being statistically significant in comparison to fibroblasts. Of note DON caused a moderate but significant viability reduction in the primary fibroblasts $(23.8 \%)$. Orlistat was able to decrease cell viability in all the cell lines, although the difference was not statistically significant for MDA-MB-231, HeLa and U-2OS cell lines. The less sensitive cells (HeLa) had an almost identical reduction in viability as compared to 9.8 and $9.5 \%$ in fibroblasts, respectively. It was noteworthy that, orlistat reached almost a total cell viability inhibition of AGS gastric cells, 95.5\% ( $\mathrm{P}=0.006)$.

Cell viability inhibition using the triple combination. As shown in Fig. 4, the combination of the three drugs led to a marked reduction in cell viability exceeding $75 \%$ inhibition in all but the pancreatic cancer cell line PANC-1, in which only a $60 \%$ reduction was observed. The triple drug combination treatment had a higher effect than treatment with any single drug, with the exception of cell lines, such as AGS (1.9 vs. $4.46 \%$ with orlistat) and SW480 (20.08 vs. $24.5 \%$ with DON) where the effect of one drug was almost the same as that of the triple combination effect. By contrast, T24 exerted a much higher cytotoxic effect with the combination of $93 \%$, compared with DON $35 \%$, lonidamine $21 \%$ and orlistat $32.3 \%$. Of utmost importance, blocking these three 'basic' metabolic pathways under our experimental conditions showed a minimal but statistically significant effect upon the viability of primary fibroblasts, with only a $23.8 \%(\mathrm{P}=0.04)$ reduction, suggesting 

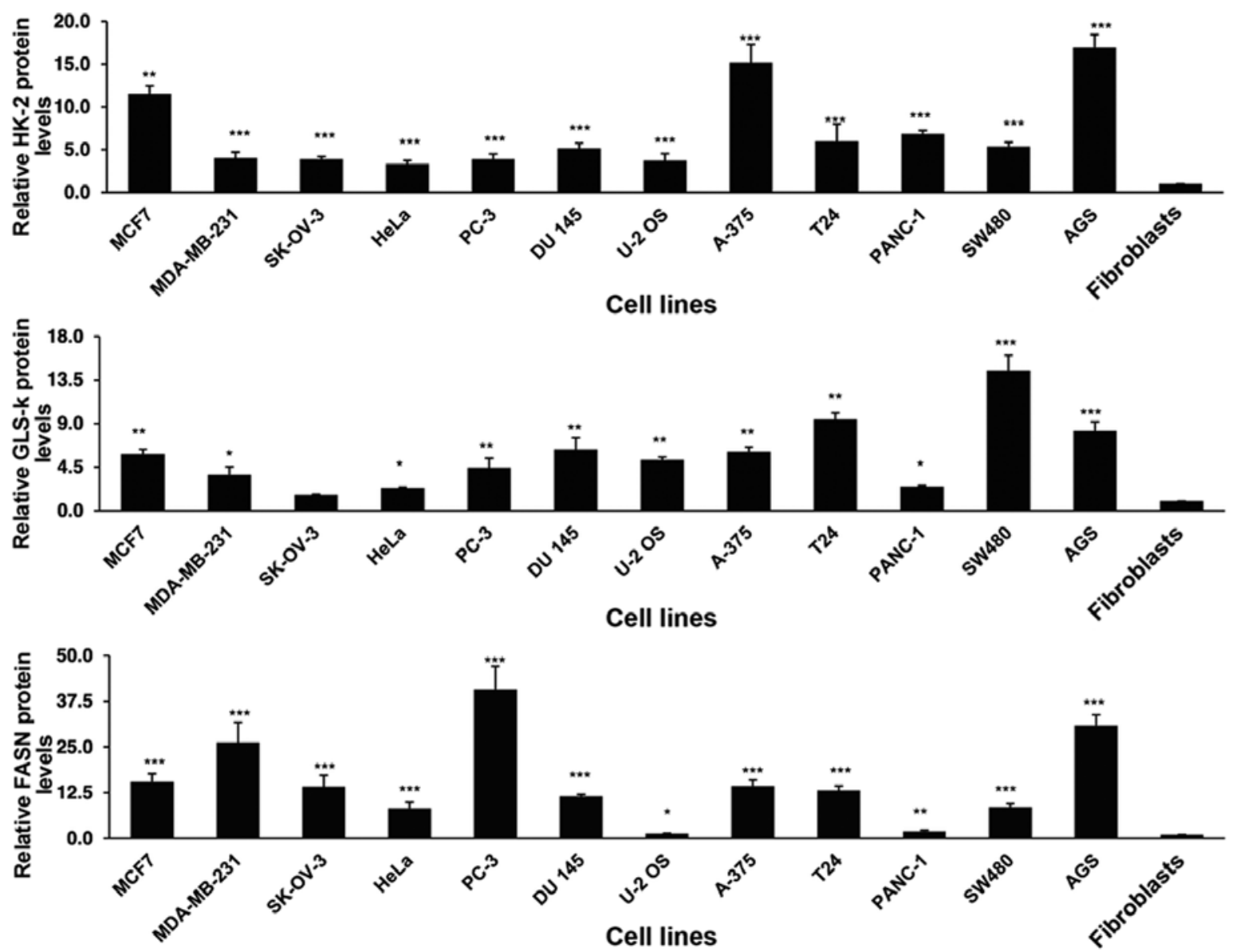

Figure 2. Protein expression of HK-2, GLS and FASN in different cell lines. Basal protein expression levels of HK-2, GLS and FASN were measured by analyses of immunofluorescence images with ImageJ. The results are expressed in units relative to the protein expression level of primary fibroblasts as normal controls. Data are presented as means \pm SD of two independent experiments. Significant differences between fibroblasts and the different malignant cell lines are indicated as ${ }^{*} \mathrm{P}<0.05,{ }^{* *} \mathrm{P}<0.01$ and ${ }^{* * *} \mathrm{P}<0.001$. The malignant cell lines overexpressed the proteins at variable degrees as compared to the non-cancer cells. HK-2, hexokinase-2; GLS, glutaminase; FASN, fatty acid synthase.

Table I. Effects of the monotherapy treatment with lonidamine, DON or orlistat on the SW480 colon cancer cell line.

\begin{tabular}{|c|c|c|c|c|c|c|}
\hline Cell line & $\begin{array}{l}\text { Lonidamine } \\
\qquad(\mu \mathrm{M})\end{array}$ & $\begin{array}{c}\mathrm{Fa} \\
\text { (Lonidamine) }\end{array}$ & $\begin{array}{l}\text { DON } \\
(\mu \mathrm{M})\end{array}$ & $\begin{array}{c}\mathrm{Fa} \\
(\mathrm{DON})\end{array}$ & $\begin{array}{c}\text { Orlistat } \\
(\mu \mathrm{M})\end{array}$ & $\begin{array}{c}\mathrm{Fa} \\
\text { (orlistat) }\end{array}$ \\
\hline \multirow[t]{5}{*}{ SW480 } & 50 & 0.4069 & 15 & 0.4492 & 17.5 & 0.2629 \\
\hline & 100 & 0.4257 & 30 & 0.7286 & 35 & 0.6382 \\
\hline & 200 & 0.6336 & 60 & 0.7860 & 70 & 0.6841 \\
\hline & 300 & 0.7519 & 90 & 0.8156 & 105 & 0.8027 \\
\hline & 400 & 0.8662 & 120 & 0.8695 & 140 & 0.9241 \\
\hline
\end{tabular}

Drug concentration is expressed in $\mu \mathrm{M}$ and fraction affected (Fa) (death cell fraction) after $48 \mathrm{~h}$ is presented as the mean of three independent experiments. DON, 6-diazo-5-oxo-L-norleucine.

that using a 'full blockade' with these drugs can be feasible in vivo.

Synergy of the triple combination. We determined whether the triple combination of lonidamine, DON and orlistat exerted a synergistic, antagonistic, or additive effect using the ChouTalalay method. For this purpose, the SW480 cell line was treated with different concentrations of each drug (Table I) and the triple combination, always under the IC50 of the drugs. As shown in Fig. 5 left panel, lonidamine, DON and orlistat in combination had a synergistic effect $(\mathrm{CI}<1)$ measured based on cell viability after 48 -h treatment. The right panel shows the comparison between the single and triple combination treatments. All of our experiments had a linear correlation coefficient (r) of the median-effect plot $>0.90$, which indicates good conformity of the data. 

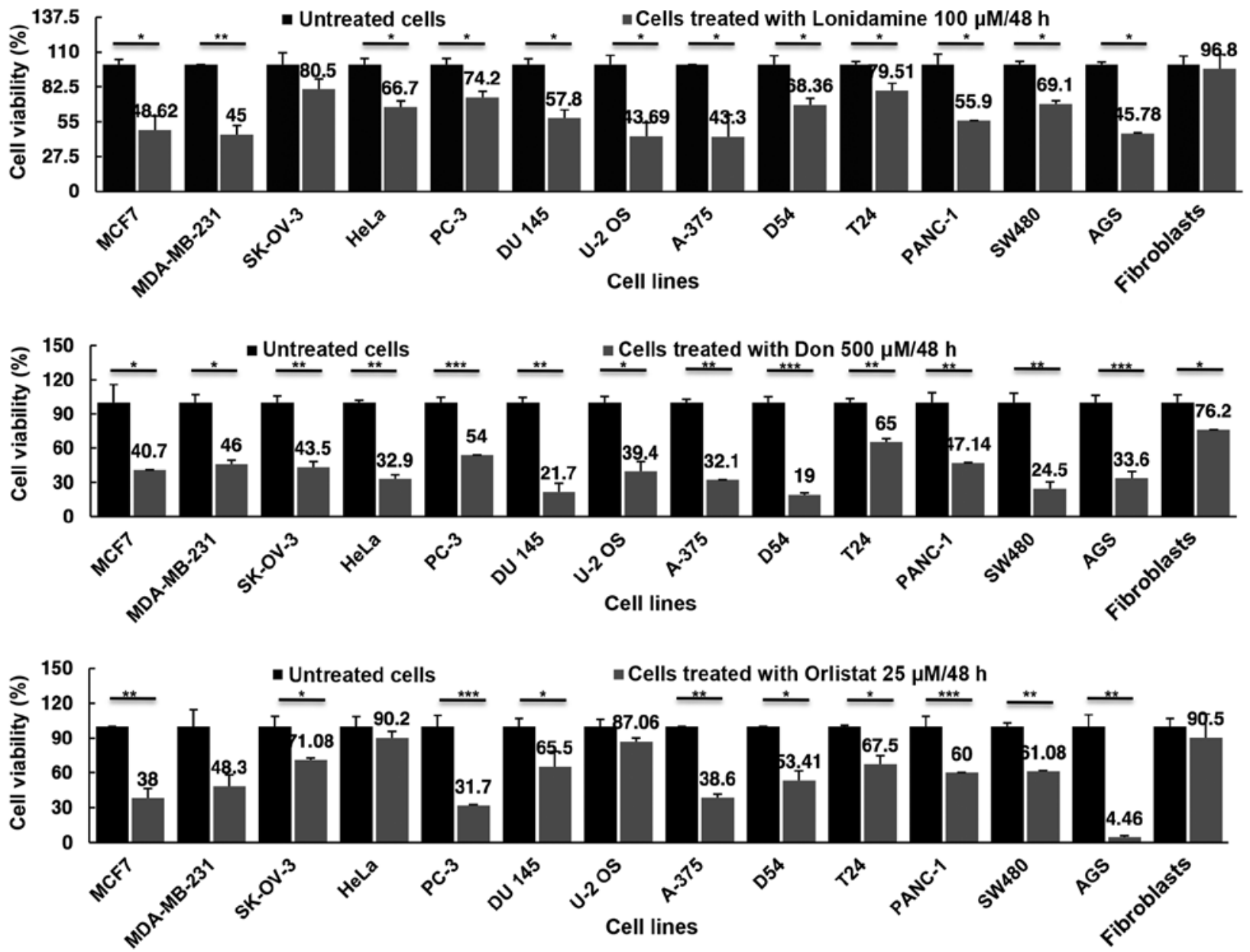

Figure 3. Cell viability inhibition by lonidamine, DON and orlistat as single drugs. Cell lines were treated with $100 \mu \mathrm{M}$ lonidamine, $500 \mu \mathrm{M}$ DON, or $25 \mu \mathrm{M}$ orlistat for $48 \mathrm{~h}$. DON treatment significantly decreased cell viability in all the cell lines. Lonidamine and orlistat had different degrees of cytotoxicity among the different cell lines. Notably, orlistat reduced $>95 \%$ cell viability in AGS cells. The effect of lonidamine and orlistat on the primary fibroblasts was not statistically significant, DON had a significant effect on fibroblasts. Data are presented as means \pm SD from three independent experiments. Significant differences between controls (vehicle) and cells treated with the drugs are indicated as ${ }^{*} \mathrm{P}<0.05,{ }^{* * *} \mathrm{P}<0.01$ and ${ }^{* * * *} \mathrm{P}<0.001$. DON, 6-diazo-5-oxo-L-norleucine.

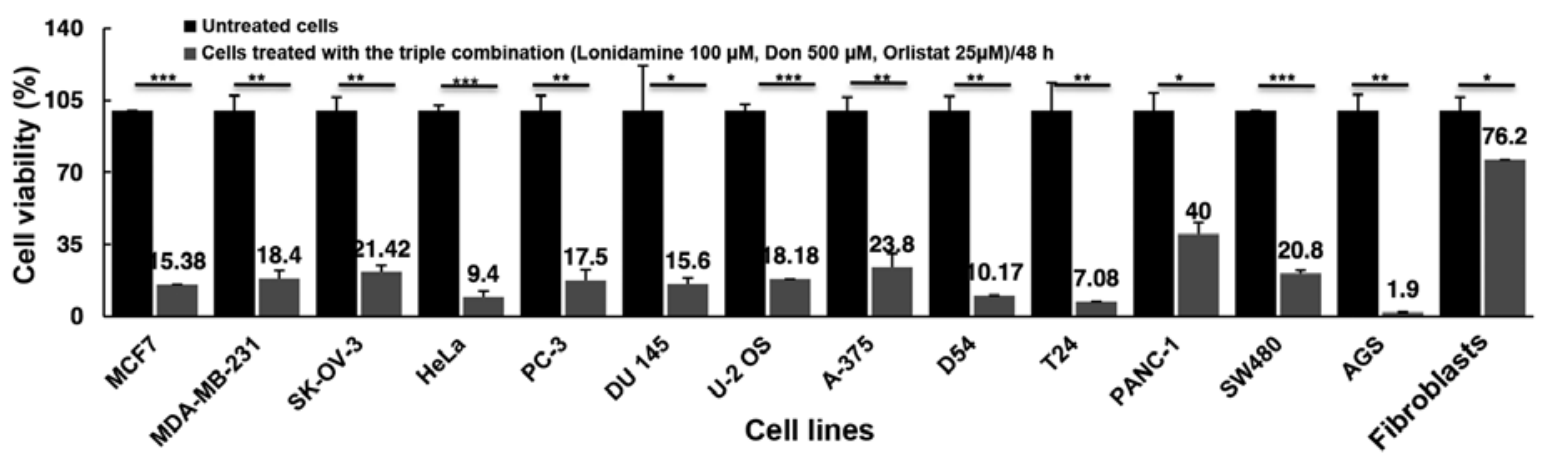

Figure 4. Effect of the triple combination on cell viability in different cell lines. All the cell lines were exposed to the triple combination (100 $\mu \mathrm{M}$ lonidamine, $500 \mu \mathrm{M}$ DON and $25 \mu \mathrm{M}$ orlistat) for $48 \mathrm{~h}$. The treatment decrease in cell viability was highly significant in most of the malignant cell lines. Primary fibroblasts had limited reduction of viability even though it was significant. Data are presented as means \pm SD from three independent experiments. P-values are a comparison between non-treated cells and treated cells, ${ }^{*} \mathrm{P}<0.05,{ }^{* *} \mathrm{P}<0.01$ and ${ }^{* * *} \mathrm{P}<0.001$. DON, 6-diazo-5-oxo-L-norleucine.

siRNA silencing of target genes. To investigate to what extent the knockdown of HK-2, GLS or FASN led to cell viability inhibition, the gene expression was individually suppressed. Results in Fig. 6 show that for HK-2 we were able to downregulate almost by half the expression of this gene, which led to $21.8 \%$ reduction on viability. The results of the treatment with lonidamine on viability were very similar to the effects of the HK-2 knocked down cells. For GLS gene, the suppression was $>50 \%$ and the reduction on viability was $46.3 \%$. However, DON led to an important reduction in cell viability with and without GLS depletion. FASN was also downregulated $>65 \%$ and induced only a small reduction on cell viability. The effect 

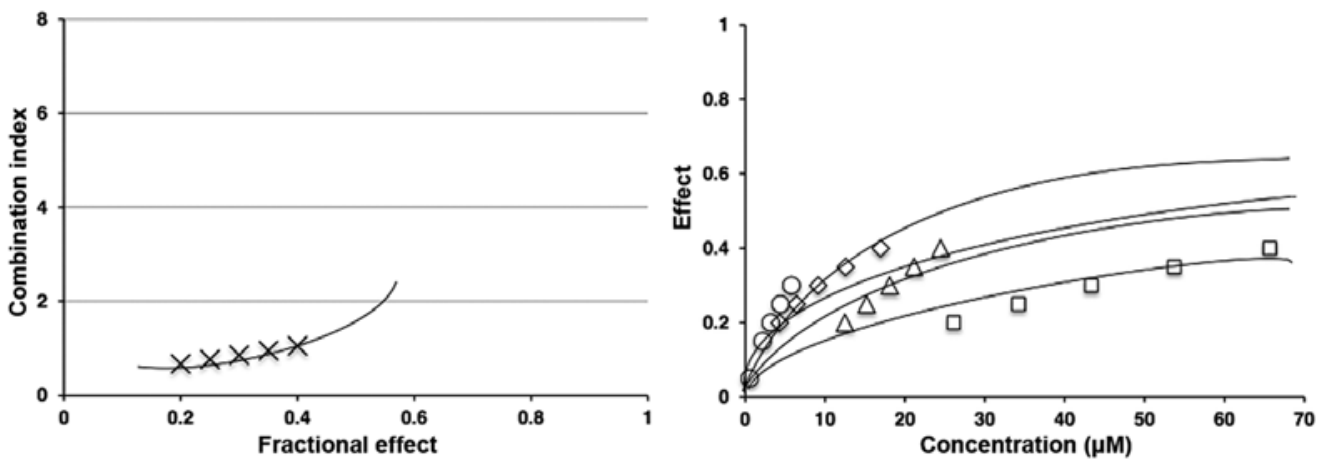

qLonidamine $O$ Don $\triangle$ Orlistat $\diamond$ Lonidamine+Don+Orlistat

Figure 5. Synergistic interaction of the triple drug combination. CalcuSyn software was used to determine the pharmacologic effect of lonidamine, DON and orlistat in combination for $48 \mathrm{~h}$. Left panel shows the combination index (CI) values for each fractional effect. Treatments with the combination at different concentrations resulted in a synergistic effect $(\mathrm{CI}<1)$. On the right panel, the drug monotherapy treatments are compared with the triple combination in a concentration-effect curve. On the left panel, CI vs. fraction-affected ( $\mathrm{Fa}$ ) plots show the synergistic effect of the triple combination at different concentrations. DON, 6-diazo-5-oxo-L-norleucine.
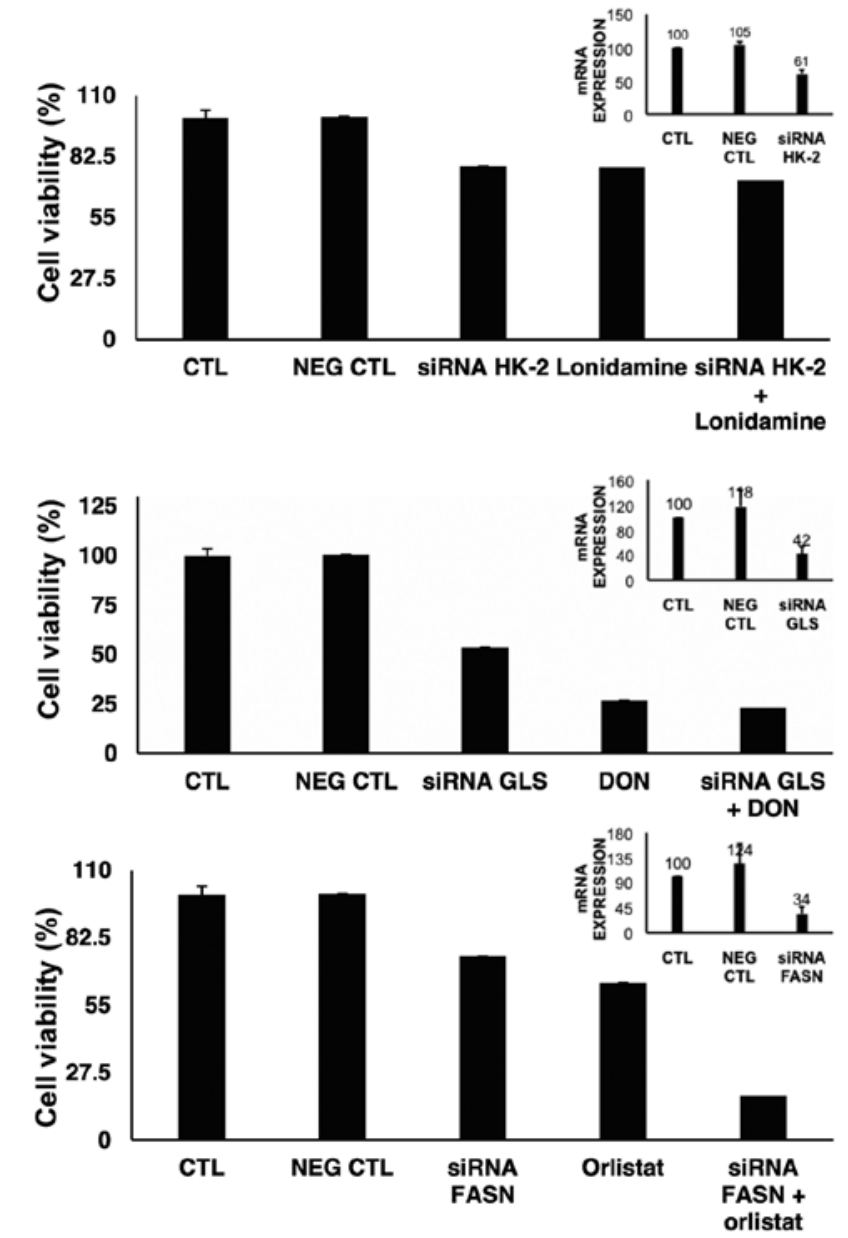

Figure 6. Knockdown of target genes and cell viability. The SW480 colon cancer cell line was used to knock down HK-2, GLS or FASN individually using siRNAs. The cells were also treated with lonidamine, DON and orlistat or the combination of lonidamine/HK-2 siRNA, DON/GLS siRNA or orlistat/FASN siRNA for $48 \mathrm{~h}$. mRNA levels of the enzymes were measured by RT-qPCR and cell viability by trypan blue dye exclusion. Untreated cells were used as controls. The cell expression levels are shown as a percentage relative to the expression level of untreated cells (right upper panel). Viability is expressed as the percentage relative to untreated cells (main panel). Data are presented as mean $\pm \mathrm{SD}$ of three independent experiments. For mRNA expression each experiment was performed in triplicate. DON, 6-diazo-5-oxo-L-norleucine; HK-2, hexokinase-2; GLS, glutaminase; FASN, fatty acid synthase.

of orlistat was slightly lower than the effect of depleted mRNA of FASN. However, cell viability was decreased when orlistat was added to FASN-depleted cells.
Administration of the triple combination in vivo. The blockage of glycolysis, glutaminolysis and de novo FA synthesis may affect normal cells in vivo. To demonstrate the feasibility of 


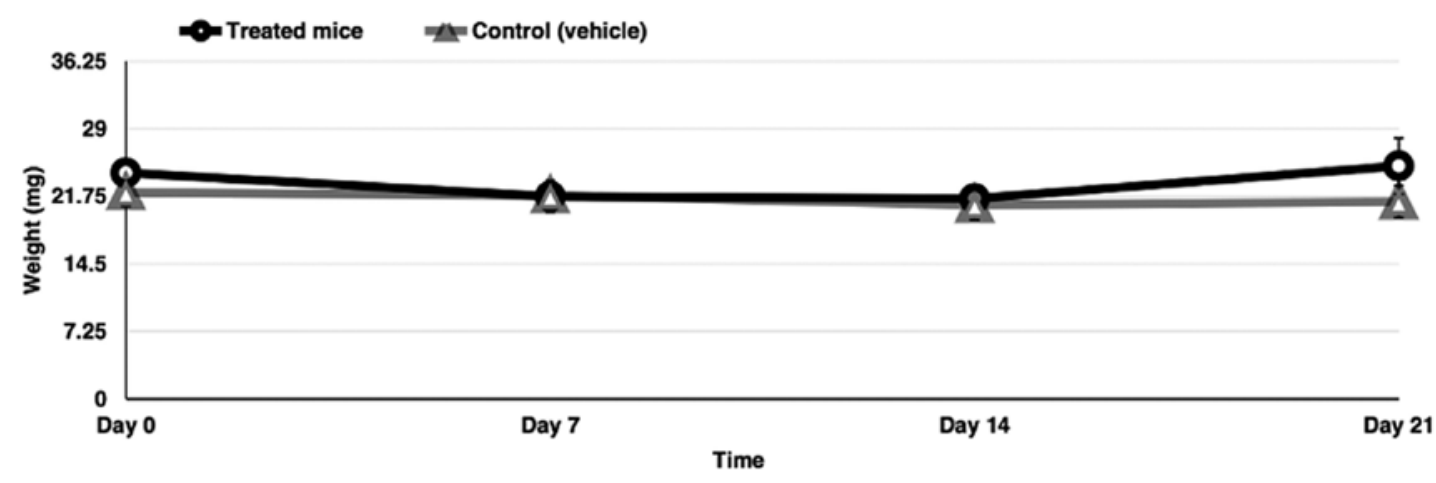

Figure 7. Feasibility of administering the triple drug combination in vivo. BALB/c female mice were treated with the triple combination (lonidamine + DON + orlistat) during 3 weeks. Body weight was measured weekly starting day 0, before treatment. There was a slight reduction of body weight during the 3 weeks, which can be related to lack of important toxic effects in the mice. Data are presented as the mean \pm SD of 6 mice. DON, 6-diazo-5-oxo-L-norleucine.

using a triple combination in vivo and based on the small effect on cell viability on primary fibroblasts, six BALB/c healthy female mice were treated with the triple combination of drugs at doses clinically achievable for 3 weeks. Notably, the mice had no significant clinical deterioration and the weight was only moderately reduced during the first and second week and then maintained or recuperated at week 4 (Fig. 7).

\section{Discussion}

The results of this study show that cancer cells as compared to primary fibroblasts have overexpression of key genes whose products are involved in the malignant metabolic phenotype. Additionally, the use of drugs targeting glycolysis, glutaminolysis and de novo synthesis of FAs or the downregulation of these target genes with siRNAs leads to a decrease on cell viability. Of note, the use of the three drugs in combination markedly increased the growth inhibitory effect on cancer cells and exerted a clear synergistic effect in vitro. As predicted by the relatively low inhibition of primary fibroblasts, the triple combination is well-tolerated in mice.

In this era of molecular-targeted therapy, most approved drugs are genotype-driven (24). Thus, drugs are directed to the product of a single genetic abnormality, while other drugs are directed to the cancer phenotype. Thus, most classical cytotoxic drugs as well as current angiogenic inhibitors are directed against the proliferative (25) and neoangiogenic phenotypes, respectively (26). The metabolic alterations of cancer cells were previously described but only recently have they been particularly investigated. Thus, the malignant metabolic phenotype is now considered another cancer hallmark (27) and many preclinical studies focusing on the development of anti-metabolic cancer agents are underway.

The three most commonly investigated metabolic alterations of cancer cells are glycolysis, glutaminolysis and the de novo synthesis of FAs. The increased activities of these pathways are therefore, natural targets to attack the malignant metabolic phenotype (28). A number of preclinical studies using drugs to target either of these pathways have demonstrated that they are effective. Among glycolytic inhibitors a high number of this drug class is being evaluated in experimental systems, reviewed in refs. 29,30. However, only lonidamine, 2-deoxy-D-glucose and dichloracetate have reached clinical trials with modest results as single agents or in combination with chemotherapy or radiation $(10,31,32)$. In particular, lonidamine the HK-2 inhibitor used in this study, has been widely investigated for the treatment of solid tumors with encouraging results in phase II-III trials for the treatment of advanced breast, ovarian and lung cancer. A review by Di Cosimo et al (10) concluded that data are insufficient to draw a firm conclusion on the efficacy of lonidamine. Thus, it seems clear that this agent merits further clinical development. Regarding glutaminolysis inhibitors, azaserine, DON and azotomycin, the three diazo analogs of L-glutamine that showed preclinical antitumor activity (33), remain unstudied as glutaminolytic agents, with the exception of a recent study in which DON was used together with recombinant GLS, showing encouraging results (34). Instead, newer selective agents against GLS are being developed. One of these new agents has recently entered into clinical phases (35). No clinical trials in cancer have been undertaken with FASN inhibitors, despite a number of these agents showed encouraging activity in several solid and hematological malignancies (13-22).

To the best of our knowledge, no preclinical studies have been performed with this triple drug combination. The most closely related study was undertaken in 1993, in which the combination of DON and 2-deoxy-D-glucose led to marked inhibition of both glutamine oxidation and glycolysis which was accompanied by increased cytotoxicity against the human TPH-1 myeloid cell line and freshly cultured myeloid blast cultures obtained from a patient (36).

The results clearly demonstrate that despite the metabolic plasticity of malignancies, most cancer cells need to overexpress these enzymes as a source of energy and precursors for macromolecule synthesis (glucose and glutamine) as well as the key enzyme to synthesize FAs, in other words, to become anabolic. In addition, the relevance of such a pattern of gene expression is shown by the fact that their pharmacological or genetic downregulation, even individually, results in growth inhibition. The variable effect of adding lonidamine, DON or orlistat on cells with depletion of the gene-coding enzyme can be explained by the fact that as expected, most drugs have off-target effects and complex diseases such as cancer may require combinatorial therapeutic approaches (37). This is most notable for orlistat where the inhibition of viability is higher when orlistat is added to FASN-downregulated cells. 
Nevertheless, the results of these experiments must be considered with caution because the efficiency of downregulation by siRNA was limited. The doses used for each individual drug to assay drug inhibition were those used in other studies and/ or taken from clinical studies of DON and lonidamine where the plasma concentration in patients was determined $(10,33)$. However, the most interesting fact is that the assays used to determine the pharmacological interaction among them showed that they are highly synergistic, particularly with lower doses of each of them. Nevertheless, these are in vitro assays thus an in vivo pharmacological interaction should be performed to confirm the results.

The results are of relevance in the field of drug repositioning for cancer therapy. As stated above, DON and lonidamine were previously clinically tested, mostly when knowledge on the metabolic alterations of cancers was limited $(10,33)$. In fact, these drugs were used as a single agent or in combination with cytotoxic drugs and overall, they were well tolerated. Orlistat, an oral drug, has never been used systemically in humans but data from literature indicate that its systemic absorption is almost none. In a study, it was found that long-term oral administration of this drug reaches $<10 \mathrm{ng} / \mathrm{ml}$ or $0.02 \mu \mathrm{M}$ in plasma (38). A case report on a child who ingested a massive dose of oral orlistat showed no systemic toxicity (39), suggesting that its systemic administration was tolerable. Our results of the cultured cells indicated that in general, primary fibroblasts are less inhibited than cancer cell lines with any of these drugs or the combination. In addition, the triple combination administered in normal BALB/c mice at doses clinically achievable do not induce major weight loss, suggesting that the repositioning of these drugs for cancer treatment as a combination is feasible.

Although in this study we did not analyze enzymatic inhibition of these drugs, its inhibitory effect on each of the enzymes mentioned has been previously demonstrated. Therefore, we can state that a 'full blockade' of the three most characterized metabolic pathways observed in cancer has promising antitumor activity in vitro and the treatment is well tolerated in vivo. As cancer cells are able to reprogramme their metabolism depending on nutrient availability $(40,41)$, more studies are needed on how cells respond to metabolic pathway inhibition. In addition, antitumor in vivo models of the combination should be undertaken.

In conclusion, to the best of our knowledge, our results demonstrate, for the first time, that cancer cells in general simultaneously overexpressed three key glycolytic, glutaminolytic and FA synthesis enzymes and that their concurrent inhibition in vitro has potent antitumor activity and synergy, suggesting that a full blockade of these metabolic alterations may be feasible and should be pursued with re-purposed or novel drugs.

\section{Acknowledgements}

Diana Cervantes-Madrid was a PhD student in the Programa de Doctorado en Ciencias Biomédicas, Universidad Nacional Autónoma de México, and received a scholarship from Consejo Nacional de Ciencia y Tecnología, Conacyt, México (245314), from Programa de Apoyo a los Estudios de Posgrado (PAEPUNAM) and the Programa de Movilidad Internacional de
Estudiantes of the Dirección General de Estudios de Posgrado (DGEP-UNAM). This work was supported by CONACyT grant no. 140654. We also like to thank Dr Victor Ruiz-López and Dr Marcela Lizano-Soberon for their support and guidance in this project.

\section{References}

1. Chen JQ and Russo J: Dysregulation of glucose transport, glycolysis, TCA cycle and glutaminolysis by oncogenes and tumor suppressors in cancer cells. Biochim Biophys Acta 1826: 370-384, 2012.

2. Levine AJ and Puzio-Kuter AM: The control of the metabolic switch in cancers by oncogenes and tumor suppressor genes. Science 330: 1340-1344, 2010.

3. Barger JF and Plas DR: Balancing biosynthesis and bioenergetics: Metabolic programs in oncogenesis. Endocr Relat Cancer 17: R287-R304, 2010.

4. Newsholme EA, Crabtree B and Ardawi MS: The role of high rates of glycolysis and glutamine utilization in rapidly dividing cells. Biosci Rep 5: 393-400, 1985.

5. McKeehan WL: Glycolysis, glutaminolysis and cell proliferation. Cell Biol Int Rep 6: 635-650, 1982.

6. Menendez JA, Ropero S, Mehmi I, Atlas E, Colomer R and Lupu R: Overexpression and hyperactivity of breast cancer-associated fatty acid synthase (oncogenic antigen-519) is insensitive to normal arachidonic fatty acid-induced suppression in lipogenic tissues but it is selectively inhibited by tumoricidal $\alpha$-linolenic and $\gamma$-linolenic fatty acids: A novel mechanism by which dietary fat can alter mammary tumorigenesis. Int J Oncol 24: 1369-1383, 2004.

7. Wang Y, Jones Voy B, Urs S, Kim S, Soltani-Bejnood M, Quigley N, Heo YR, Standridge M, Andersen B, Dhar M, et al: The human fatty acid synthase gene and de novo lipogenesis are coordinately regulated in human adipose tissue. J Nutr 134: 1032-1038, 2004.

8. Little JL and Kridel SJ: Fatty acid synthase activity in tumor cells. Subcell Biochem 49: 169-194, 2008.

9. Menendez JA and Lupu R: Fatty acid synthase and the lipogenic phenotype in cancer pathogenesis. Nat Rev Cancer 7: 763-777, 2007.

10. Di Cosimo S, Ferretti G, Papaldo P, Carlini P, Fabi A and Cognetti F: Lonidamine: Efficacy and safety in clinical trials for the treatment of solid tumors. Drugs Today 39: 157-174, 2003.

11. Kisner DL, Catane R and Muggia FM: The rediscovery of DON (6-diazo-5-oxo-L-norleucine). Recent Results Cancer Res 74: 258-263, 1980.

12. Lupu R and Menendez JA: Pharmacological inhibitors of Fatty Acid Synthase (FASN) - catalyzed endogenous fatty acid biogenesis: A new family of anti-cancer agents? Curr Pharm Biotechnol 7: 483-493, 2006.

13. Menendez JA, Vellon L and Lupu R: Antitumoral actions of the anti-obesity drug orlistat $\left(\mathrm{Xenical}^{\mathrm{TM}}\right)$ in breast cancer cells: Blockade of cell cycle progression, promotion of apoptotic cell death and PEA3-mediated transcriptional repression of Her $2 / n e u$ (erbB-2) oncogene. Ann Oncol 16: 1253-1267, 2005

14. Yang CS, Matsuura K, Huang NJ, Robeson AC, Huang B, Zhang L and Kornbluth S: Fatty acid synthase inhibition engages a novel caspase- 2 regulatory mechanism to induce ovarian cancer cell death. Oncogene 34: 3264-3272, 2015.

15. Fujiwara J, Sowa Y, Horinaka M, Koyama M, Wakada M, Miki T and Sakai T: The anti-obesity drug orlistat promotes sensitivity to TRAIL by two different pathways in hormone-refractory prostate cancer cells. Int J Oncol 40: 1483-1491, 2012.

16. Zecchin KG, Rossato FA, Raposo HF, Melo DR, Alberici LC, Oliveira HC, Castilho RF, Coletta RD, Vercesi AE and Graner E: Inhibition of fatty acid synthase in melanoma cells activates the intrinsic pathway of apoptosis. Lab Invest 91: 232-240, 2011.

17. Samudio I, Harmancey R, Fiegl M, Kantarjian H, Konopleva M, Korchin B, Kaluarachchi K, Bornmann W, DuvvuriS, Taegtmeyer H, et al: Pharmacologic inhibition of fatty acid oxidation sensitizes human leukemia cells to apoptosis induction. J Clin Invest 120: 142-156, 2010.

18. Kant S, Kumar A and Singh SM: Tumor growth retardation and chemosensitizing action of fatty acid synthase inhibitor orlistat on T cell lymphoma: Implication of reconstituted tumor microenvironment and multidrug resistance phenotype. Biochim Biophys Acta 1840: 294-302, 2014. 
19. Tirado-Vélez JM, Joumady I, Sáez-Benito A, Cózar-Castellano I and Perdomo G; Tirado-Vélez JM: Inhibition of fatty acid metabolism reduces human myeloma cells proliferation. PLoS One 7: e46484, 2012

20. Olsen AM, Eisenberg BL, Kuemmerle NB, Flanagan AJ, Morganelli PM, Lombardo PS, Swinnen JV and Kinlaw WB: Fatty acid synthesis is a therapeutic target in human liposarcoma. Int J Oncol 36: 1309-1314, 2010.

21. Dowling S, Cox J and Cenedella RJ: Inhibition of fatty acid synthase by Orlistat accelerates gastric tumor cell apoptosis in culture and increases survival rates in gastric tumor bearing mice in vivo. Lipids 44: 489-498, 2009.

22. Chuang HY, Chang YF and Hwang JJ: Antitumor effect of orlistat, a fatty acid synthase inhibitor, is via activation of caspase- 3 on human colorectal carcinoma-bearing animal. Biomed Pharmacother 65: 286-292, 2011.

23. Pardo A, Selman M, Ramírez R, Ramos C, Montaño M, Stricklin G and Raghu G: Production of collagenase and tissue inhibitor of metalloproteinases by fibroblasts derived from normal and fibrotic human lungs. Chest 102: 1085-1089, 1992.

24. Huang RS and Ratain MJ: Pharmacogenetics and pharmacogenomics of anticancer agents. CA Cancer J Clin 59: 42-55, 2009.

25. Fernandes DJ, Sur P, Kute TE and Capizzi RL: Proliferation-dependent cytotoxicity of methotrexate in murine L5178Y leukemia. Cancer Res 48: 5638-5644, 1988

26. Gatto B and Cavalli M: From proteins to nucleic acid-based drugs: The role of biotech in anti-VEGF therapy. Anticancer Agents Med Chem 6: 287-301, 2006.

27. Hanahan D and Weinberg RA: Hallmarks of cancer: The next generation. Cell 144: 646-674, 2011

28. Zhao Y, Liu H, Riker AI, Fodstad O, Ledoux SP, Wilson GL and Tan M: Emerging metabolic targets in cancer therapy. Front Biosci 16: 1844-1860, 2011

29. Ganapathy-Kanniappan S and Geschwind JF: Tumor glycolysis as a target for cancer therapy: Progress and prospects. Mol Cancer 12: 152, 2013.

30. Granchi $\mathrm{C}$ and Minutolo F: Anticancer agents that counteract tumor glycolysis. ChemMedChem 7: 1318-1350, 2012.

31. Dwarakanath BS, Singh D, Banerji AK, Sarin R, Venkataramana NK, Jalali R, Vishwanath PN, Mohanti BK, Tripathi RP, Kalia VK, et al: Clinical studies for improving radiotherapy with 2-deoxy-D-glucose: Present status and future prospects. J Cancer Res Ther 5 (Suppl 1): S21-S26, 2009.
32. Garon EB, Christofk HR, Hosmer W, Britten CD, Bahng A, Crabtree MJ, Hong CS, Kamranpour N, Pitts S, Kabbinavar F, et al: Dichloroacetate should be considered with platinum-based chemotherapy in hypoxic tumors rather than as a single agent in advanced non-small cell lung cancer. J Cancer Res Clin Oncol 140: 443-452, 2014.

33. Catane R, Von Hoff DD, Glaubiger DL and Muggia FM: Azaserine, DON and azotomycin: Three diazo analogs of L-glutamine with clinical antitumor activity. Cancer Treat Rep 63: 1033-1038, 1979.

34. Unger C, Mueller C, Bausch MP, et al: A phase I schedule optimization study of pegylatedglutaminase (PEG-PGA) plus 6-diazo-5-oxo-1-norleucine (DON) in patients (pts) with advanced solid tumors. J Clin Oncol 29: abs. 3049, 2011.

35. Strickland DK: Study of the glutaminase inhibitor CB-839 in solid tumors. Clinical Trials Identifier: NCT02071862. February 2014. http://clinicaltrials.gov/ct2/results?term=glutami nasecancer\&Search=Search. Last updated March 9, 2015.

36. Griffiths M, Keast D, Patrick G, Crawford M and Palmer TN: The role of glutamine and glucose analogues in metabolic inhibition of human myeloid leukaemia in vitro. Int J Biochem 25: 1749-1755, 1993.

37. Anighoro A, Bajorath J and Rastelli G: Polypharmacology: Challenges and opportunities in drug discovery. J Med Chem 57: 7874-7887, 2014

38. Zhi J, Mulligan TE and Hauptman JB: Long-term systemic exposure of orlistat, a lipase inhibitor and its metabolites in obese patients. J Clin Pharmacol 39: 41-46, 1999.

39. O'Connor MB: An orlistat 'overdose' in a child. Ir J Med Sci 179: $315,2010$.

40. Young CD and Anderson SM: Sugar and fat - that's where it's at: Metabolic changes in tumors. Breast Cancer Res 10: 202, 2008.

41. Sankaranarayanapillai M, Zhang N, Baggerly KA and Gelovani JG: Metabolic shifts induced by fatty acid synthase inhibitor orlistat in non-small cell lung carcinoma cells provide novel pharmacodynamic biomarkers for positron emission tomography and magnetic resonance spectroscopy. Mol Imaging Biol 15: 136-147, 2013 